01

\title{
Общие свойства ядер интеграла столкновений нелинейного уравнения Больцмана
}

\author{
(С Л.А. Бакалейников, Е.Ю. Флегонтова, Э.А. Тропп \\ Физико-технический институт им. А.Ф.Иоффре РАН, \\ 194021 Санкт-Петербург, Россия \\ e-mail: fl.xiees@mail.ioffe.ru
}

Поступило в Редакцию 22 февраля 2019 г.

В окончательной редакции 22 февраля 2019 г.

Принято к публикации 5 марта 2019 г.

Рассмотрены общие не зависящие от сечения рассеяния свойства ядер интеграла обратных столкновений, а также ядер интегральных операторов, возникающих при разложении интеграла столкновений по сферическим гармоникам. Полученные ранее рекуррентные соотношения между ядрами обобщены на случай взаимодействия частиц разных масс. Показано, что найденные свойства обеспечивают аддитивную инвариантность числа частиц, энергии и импульса.

Ключевые слова: кинетическая теория, нелинейное уравнение Больцмана, интеграл столкновений.

DOI: $10.21883 / J T F .2019 .10 .48162 .66-19$

\section{Введение}

Основной трудностью при численном решении кинетического уравнения Больцмана, даже на современном уровне развития вычислительной техники, остается расчет интеграла столкновений, который требует пятикратного интегрирования по сложной области. Широко известным подходом к решению этой задачи является моментный метод, в котором кинетическое уравнение раскладывается по функциям Барнетта [1-3]. При этом расчет интеграла столкновений сводится к алгебраическим операциям с числовыми константами матричными элементами интеграла столкновений. Было установлено $[4,5]$, что между матричными элементами существуют связи, которые позволяют последовательно находить их с помощью рекуррентной процедуры [6-9].

При использовании моментного метода возникают ограничения на сходимость функции распределения $(Ф Р)$, которые можно устранить, раскладывая лишь угловую зависимость ФР и кинетического уравнения по сферическим гармоникам. В этом случае интеграл столкновений заменяется набором более простых интегральных операторов, ядра которых представляют собой проекции ядра интеграла столкновений на сферические гармоники и зависят лишь от модулей скоростей взаимодействующих частиц. Эти ядра полностью определяются сечением взаимодействия частиц и могут использоваться по аналогии с матричными элементами интеграла столкновений.

Использование предварительно рассчитанных ядер интеграла столкновений существенно облегчает процесс численного решения уравнения Больцмана. В то же время построение ядер интеграла столкновений представляет собой самостоятельную сложную задачу. Исследованию и построению этих ядер посвящен ряд наших предыдущих работ. В работах $[10,11]$ показано, что в случае взаимодействия частиц одного сорта ядра интеграла столкновений, так же как и матричные элементы, связаны рекуррентными соотношениями. В работе [12] предложена рекуррентная процедура построения ядер с последовательно возрастающими индексами (индексы ядер соответствуют индексам сферических гармоник, на которые проецируется интеграл столкновений).

Часто интеграл столкновений может быть разделен на интегралы прямых и обратных столкновений, описывающие приход частиц в рассматриваемый элемент фазового объема и уход из него. В работе [13] мы изучили ряд свойств ядер интеграла прямых столкновений. В настоящей работе исследуются некоторые общие свойства ядер, которые являются отражением симметрии парных столкновений и законов сохранения при взаимодействии частиц. Некоторые из этих свойств в изотропном случае были рассмотрены в работе [14]. Мы также обобщим полученные в [10] соотношения между ядрами на случай взаимодействия частиц разных сортов. Будет показано, что полученные свойства ядер обеспечивают выполнение законов сохранения числа частиц, импульса и энергии.

Рассмотрим газ, состоящий из частиц разных сортов. Скорость изменения числа частиц сорта $a$ в единице фазового объема за счет взаимодействия с частицами сорта $b$ можно записать в виде

$$
\left.\left(\frac{\partial n_{a} f_{a}}{\partial t}\right)\right|_{c o l} ^{(a, b)}=n_{a} n_{b} \hat{I}\left(f_{a}, f_{b}\right) .
$$

Здесь $f_{a}, f_{b}$ - нормированные на единицу функции распределения частиц сортов $a$ и $b, n_{a}, n_{b}$ - их концентрации, $\hat{I}\left(f_{a}, f_{b}\right)$ - интеграл столкновений. Как известно, при произвольном степенном потенциале взаимодействия сечение рассеяния обладает неинтегрируемой 
особенностью при нулевом угле рассеяния. Тем не менее для всех степенных потенциалов жестче кулоновского интеграл столкновений конечен, и вклад в него от малых углов рассеяния мал [15]. Поэтому интеграл столкновений с точностью до малой поправки может быть представлен в виде разности интегралов обратных $\left(\hat{I}^{+}\left(f_{a}, f_{b}\right)\right)$ и прямых $\left(\hat{I}^{-}\left(f_{a}, f_{b}\right)\right)$ столкновений

$$
\hat{I}\left(f_{a}, f_{b}\right)=\hat{I}^{+}\left(f_{a}, f_{b}\right)-\hat{I}^{-}\left(f_{a}, f_{b}\right) .
$$

При вычислении $\hat{I}^{+}\left(f_{a}, f_{b}\right), \hat{I}^{-}\left(f_{a}, f_{b}\right)$ интегрирование проводится до некоторого малого угла рассеяния. Дифференциальное сечение при этом полагается равным нулю в малой окрестности нулевого угла рассеяния, и полное сечение можно считать конечным.

Интеграл столкновений представляет собой билинейный интегральный оператор и может быть записан в виде

$$
\hat{I}\left(f_{a}, f_{b}\right)=\iint G\left(\mathbf{v}, \mathbf{v}_{1}, \mathbf{v}_{2}\right) f_{a}\left(\mathbf{v}_{1}\right) f_{b}\left(\mathbf{v}_{2}\right) d \mathbf{v}_{1} d \mathbf{v}_{2},
$$

где ядро $G\left(\mathbf{v}, \mathbf{v}_{1}, \mathbf{v}_{2}\right)$ зависит от векторных скоростей и в соответствии с (2) может быть представлено как разность ядер интегралов обратных $G^{+}\left(\mathbf{v}, \mathbf{v}_{1}, \mathbf{v}_{2}\right)$ и прямых $G^{-}\left(\mathbf{v}, \mathbf{v}_{1}, \mathbf{v}_{2}\right)$ столкновений:

$$
G\left(\mathbf{v}, \mathbf{v}_{1}, \mathbf{v}_{2}\right)=G^{+}\left(\mathbf{v}, \mathbf{v}_{1}, \mathbf{v}_{2}\right)-G^{-}\left(\mathbf{v}, \mathbf{v}_{1}, \mathbf{v}_{2}\right) .
$$

Перейдем в (1) к безразмерным переменным, выбирая, как в $[13,16]$, в качестве масштабов концентрации и скорости средние концентрации $n_{a}^{0}, n_{b}^{0}$ и тепловые скорости $v_{T_{a}}=\sqrt{2 k T / m_{a}}, \quad v_{T_{b}}=\sqrt{2 k T / m_{b}}$ частиц сортов $a$ и $b$, а в качестве масштаба времени - обратную частоту столкновений $\tau_{T}^{a b}=l_{a b} / v_{T_{a}}$, где $l_{a b}=\left(\sqrt{2} n_{b}^{0} \Sigma_{e f f}^{a b}(T)\right)^{-1}$ - длина свободного пробега. Здесь $T$ - средняя температура газа, $m_{a}, m_{b}$ - массы частиц $a$ и $b$, эффективное сечение $\Sigma_{e f f}^{a b}(T)$ определяется как сечение для такого газа из твердых шаров, вязкость которого равна вязкости газа с данным молекулярным потенциалом. Приведенные в [17,18] выражения для вязкости газа из твердых шаров и газа, в котором взаимодействие описывается степенным потенциалом с показателем степени $\kappa, \varphi_{a b}(r)=\alpha\left(d_{a b} / 2 r\right)^{\kappa}$, дают

$$
\Sigma_{e f f}^{a b}(T)=\pi\left(d_{a b}\right)^{2}\left(\frac{A_{2}(\kappa+1) \Gamma(4-2 / \kappa)}{8}\right)\left(\frac{k T}{\alpha \kappa}\right)^{-2 / \kappa} .
$$

Здесь $\Gamma(z)$ - гамма-функция, а коэффициенты $A_{2}(\kappa+1)$ приведены в [17,18].

Определим безразмерные скорости

$$
\begin{gathered}
\mathbf{c}_{a}=\left(\mathbf{v}_{a}-\mathbf{u}\right) / v_{T_{a}}, \quad \mathbf{c}_{1 a}=\left(\mathbf{v}_{1 a}-\mathbf{u}\right) / v_{T_{a}}, \\
\mathbf{c}_{2 b}=\left(\mathbf{v}_{2 b}-\mathbf{u}\right) / v_{T_{b}},
\end{gathered}
$$

где $\mathbf{u}$ - средняя скорость газа. Безразмерные ФР и ядро интеграла столкновений определятся соотношениями

$$
\begin{gathered}
\tilde{f}_{a}\left(\mathbf{c}_{a}\right)=f_{a}\left(\mathbf{c}_{a} v_{T_{a}}+\mathbf{u}\right)\left(v_{T_{a}}\right)^{3}, \\
\tilde{f}_{b}\left(\mathbf{c}_{b}\right)=f_{b}\left(\mathbf{c}_{b} v_{T_{b}}+\mathbf{u}\right)\left(v_{T_{b}}\right)^{3},
\end{gathered}
$$

$$
\begin{aligned}
& \tilde{G}\left(\mathbf{c}_{a}, \mathbf{c}_{1 a}, \mathbf{c}_{2 b}\right)=\left(v_{T_{a}}\right)^{3} \tau_{T}^{a b} n_{b}^{0} G\left(\mathbf{v}_{a}, \mathbf{v}_{1 a}, \mathbf{v}_{2 b}\right) \\
& \quad=\left(v_{T_{a}}\right)^{3} \tau_{T}^{a b} n_{b}^{0} G\left(\mathbf{c}_{a} v_{T_{a}}+\mathbf{u}, \mathbf{c}_{1 a} v_{T_{a}}+\mathbf{u}, \mathbf{c}_{2 b} v_{T_{b}}+\mathbf{u}\right) .
\end{aligned}
$$

Ниже будем опускать тильду и использовать для безразмерного ядра и безразмерных функций распределения обозначения $G\left(\mathbf{c}, \mathbf{c}_{1}, \mathbf{c}_{2}\right)$ и $f_{a}\left(\mathbf{c}_{a}\right), f_{b}\left(\mathbf{c}_{b}\right)$.

При разложении по сферическим гармоникам интеграл столкновений заменяется набором более простых интегральных операторов, ядра которых зависят лишь от модулей скоростей. Как показано в $[4,11]$, эти ядра пропорциональны ядрам $G_{l_{1}, l_{2}}^{l}\left(c_{a}, c_{1 a}, c_{2 b}\right)$, представляющим собой проекцию $G\left(\mathbf{c}_{a}, \mathbf{c}_{1 a}, \mathbf{c}_{2 b}\right)$ на полиномы Лежандра $P_{l}(\cos \vartheta)$ :

$$
\begin{gathered}
G_{l_{1}, l_{2}}^{l}\left(c_{a}, c_{1 a}, c_{2 b}\right)=\frac{1}{2 \pi\left\|P_{l}\right\|^{2}} \iiint G\left(\mathbf{c}_{a}, \mathbf{c}_{1 a}, \mathbf{c}_{2 b}\right) \\
\quad \times P_{l}(\cos \vartheta) P_{l_{1}}\left(\cos \vartheta_{1}\right) P_{l_{2}}\left(\cos \vartheta_{2}\right) d \Omega d \Omega_{1} d \mathbf{\Omega}_{2}
\end{gathered}
$$

Здесь $\vartheta, \vartheta_{1}, \vartheta_{2}$ - зенитные углы скоростей $\mathbf{c}_{a}, \mathbf{c}_{1 a}, \mathbf{c}_{2 b}$ в сферической системе координат с фиксированной осью z. Согласно обобщенной теореме Гекке (ОТГ) [4], эти ядра отличны от нуля лишь при комбинациях индексов, удовлетворяющих условиям: $\left|l_{1}-l_{2}\right| \leq l \leq l_{1}+l_{2}$, $l+l_{1}+l_{2}$ - четное число. В дальнейшем без потери общности будем рассматривать осесимметричный случай. С использованием ядер $G_{l_{1}, l_{2}}^{l}\left(c_{a}, c_{1 a}, c_{2 b}\right)$ интеграл столкновений (3) запишется в виде

$$
\begin{aligned}
& \left(v_{T_{a}}\right)^{3} \tau_{T}^{a b} n_{b}^{0} \hat{I}\left(f_{a}, f_{b}\right)=\sum_{l, l_{1}, l_{2}} P_{l}\left(x_{a}\right) \int_{0}^{\infty} \int_{0}^{\infty} G_{l_{1}, l_{2}}^{l}\left(c_{a}, c_{1 a}, c_{2 b}\right) \\
& \times f_{l_{1}}^{a}\left(c_{a 1}\right) f_{l_{2}}^{b}\left(c_{b 2}\right)\left(c_{a 1}\right)^{2} d c_{a 1}\left(c_{b 2}\right)^{2} d c_{b 2},
\end{aligned}
$$

где $f_{l}^{a}\left(c_{a}\right), f_{l}^{b}\left(c_{b}\right)$ - коэффициенты разложения ФР $f_{a}\left(\mathbf{c}_{a}\right), f_{b}\left(\mathbf{c}_{b}\right)$ по полиномам Лежандра.

\section{Свойства симметрии ядер в случае взаимодействия частиц одного сорта}

Рассмотрим свойства ядер в случае, когда взаимодействуют частицы одного сорта. При этом индексы сорта частиц в обозначении величин будем опускать. Для вывода свойств ядер в этом случае удобно использовать введенную в [19] функцию $W\left(\mathbf{v}_{1}, \mathbf{v}_{2} \mid \mathbf{v}, \mathbf{v}^{\prime}\right)$, описывающую вероятность того, что частицы со скоростями $\mathbf{v}_{1}, \mathbf{v}_{2}$ после столкновения приобретают скорости $\mathbf{v}, \mathbf{v}^{\prime}$. Согласно [19], $W\left(\mathbf{v}_{1}, \mathbf{v}_{2} \mid \mathbf{v}, \mathbf{v}^{\prime}\right)$ имеет вид

$$
\begin{gathered}
W\left(\mathbf{v}_{1}, \mathbf{v}_{2} \mid \mathbf{v}, \mathbf{v}^{\prime}\right)=\sigma\left(\left|\mathbf{v}_{1}-\mathbf{v}_{2}\right|, \arccos \left(\frac{\left(\left(\mathbf{v}_{1}-\mathbf{v}_{2}\right)\left(\mathbf{v}-\mathbf{v}^{\prime}\right)\right)}{\left|\mathbf{v}_{1}-\mathbf{v}_{2}\right|\left|\mathbf{v}-\mathbf{v}^{\prime}\right|}\right)\right) \\
\times \delta\left(\frac{\mathbf{v}_{1}+\mathbf{v}_{2}-\mathbf{v}-\mathbf{v}^{\prime}}{2}\right) \delta\left(\frac{\left(\mathbf{v}_{1}-\mathbf{v}_{2}\right)^{2}-\left(\mathbf{v}-\mathbf{v}^{\prime}\right)^{2}}{2}\right) . \quad(8)
\end{gathered}
$$

Здесь сечение рассеяния $\sigma(g, \theta)$ зависит от модуля относительной скорости сталкивающихся частиц 
$g=\left|\mathbf{v}_{1}-\mathbf{v}_{2}\right|$ и угла рассеяния $\theta=\arccos \left(\frac{\left(\left(\mathbf{v}_{1}-\mathbf{v}_{2}\right)\left(\mathbf{v}-\mathbf{v}^{\prime}\right)\right)}{\left|\mathbf{v}_{1}-\mathbf{v}_{2}\right|\left|\mathbf{v}-\mathbf{v}^{\prime}\right|}\right)$ (т.е. угла между относительными скоростями до и после взаимодействия). Дельта-функции обеспечивают выполнение законов сохранения энергии и импульса при столкновении. Для перехода к безразмерным переменным умножим (3) на $\left(v_{T}\right)^{3} \tau_{T} n^{0}$ и используем (5). Введем безразмерную функцию $\left(v_{T}\right)^{6} \tau_{T} n^{0} W\left(\mathbf{c}_{1} v_{T}+\mathbf{u}, \mathbf{c}_{2} v_{T}+\right.$ $\left.+\mathbf{u} \mid \mathbf{c} v_{T}+\mathbf{u}, \mathbf{c}^{\prime} v_{T}+\mathbf{u}\right)$, для которой оставим обозначение $W\left(\mathbf{c}_{1}, \mathbf{c}_{2} \mid \mathbf{c}, \mathbf{c}^{\prime}\right)$. Ядра $G^{+}\left(\mathbf{c}, \mathbf{c}_{1}, \mathbf{c}_{2}\right), G^{-}\left(\mathbf{c}, \mathbf{c}_{1}, \mathbf{c}_{2}\right)$ связаны с $W\left(\mathbf{c}_{1}, \mathbf{c}_{2} \mid \mathbf{c}, \mathbf{c}^{\prime}\right)$ соотношениями

$$
\begin{gathered}
G^{+}\left(\mathbf{c}, \mathbf{c}_{1}, \mathbf{c}_{2}\right)=\int W\left(\mathbf{c}_{1}, \mathbf{c}_{2} \mid \mathbf{c}, \mathbf{c}^{\prime}\right) d \mathbf{c}^{\prime}, \\
G^{-}\left(\mathbf{c}, \mathbf{c}_{1}, \mathbf{c}_{2}\right)=\delta\left(\mathbf{c}_{1}-\mathbf{c}\right) \iint W\left(\mathbf{c}_{1}, \mathbf{c}_{2} \mid \mathbf{c}_{1}^{\prime}, \mathbf{c}_{2}^{\prime}\right) d \mathbf{c}_{2}^{\prime} d \mathbf{c}_{1}^{\prime} .
\end{gathered}
$$

Ядро интеграла прямых столкновений $G^{-}\left(\mathbf{c}, \mathbf{c}_{1}, \mathbf{c}_{2}\right)$ подробно проанализировано в работе [13], поэтому основное внимание сосредоточим на свойствах ядра $G^{+}\left(\mathbf{c}, \mathbf{c}_{1}, \mathbf{c}_{2}\right)$. Явное выражение для $G^{+}\left(\mathbf{c}, \mathbf{c}_{1}, \mathbf{c}_{2}\right)$ приведено в нашей работе [20]

$$
\begin{aligned}
& G^{+}\left(\mathbf{c}, \mathbf{c}_{1}, \mathbf{c}_{2}\right)=\frac{8}{\sqrt{2} \Sigma_{e f f}(T)} \sigma\left(\left|\mathbf{c}_{1}-\mathbf{c}_{2}\right| v_{T},\right. \\
& \left.\arccos \left(\frac{\left(\left(\mathbf{c}_{1}-\mathbf{c}_{2}\right)\left(2 \mathbf{c}-\mathbf{c}_{1}-\mathbf{c}_{2}\right)\right)}{\left|\mathbf{c}_{1}-\mathbf{c}_{2}\right|\left|2 \mathbf{c}-\mathbf{c}_{1}-\mathbf{c}_{2}\right|}\right)\right) \\
& \quad \times \frac{\delta\left(\left|\mathbf{c}_{1}-\mathbf{c}_{2}\right|-\left|2 \mathbf{c}-\mathbf{c}_{1}-\mathbf{c}_{2}\right|\right)}{\left|\mathbf{c}_{1}-\mathbf{c}_{2}\right|} .
\end{aligned}
$$

Парные столкновения одинаковых частиц обладают некоторыми свойствами симметрии, что может быть использовано для получения общих свойств ядер интеграла столкновений, не зависящих от вида сечения рассеяния. Выводу таких свойств и посвящен этот раздел настоящей работы.

Прежде всего, частицы 1 и 2 можно переставить местами, при этом поменяются местами скорости $\mathbf{c}_{1}, \mathbf{c}_{2}$ и $\mathbf{c}, \mathbf{c}^{\prime}$. Поэтому для любого сечения рассеяния справедливо равенство

$$
W\left(\mathbf{c}_{2}, \mathbf{c}_{1} \mid \mathbf{c}^{\prime}, \mathbf{c}\right)=W\left(\mathbf{c}_{1}, \mathbf{c}_{2} \mid \mathbf{c}, \mathbf{c}^{\prime}\right) .
$$

Интегрируя это соотношение по с , $\mathbf{c}^{\prime}$, найдем

$$
\int G^{+}\left(\mathbf{c}, \mathbf{c}_{2}, \mathbf{c}_{1}\right) d \mathbf{c}=\int G^{+}\left(\mathbf{c}, \mathbf{c}_{1}, \mathbf{c}_{2}\right) d \mathbf{c} .
$$

Соответственно для ядер, зависящих от модулей скорости, имеем

$$
\int G_{i, i}^{0^{+}}\left(c, c_{2}, c_{1}\right) c^{2} d c=\int G_{i, i}^{0^{+}}\left(c, c_{1}, c_{2}\right) c^{2} d c
$$

для любых значений индекса $i$. Здесь учтено, что $d \mathbf{c}=c^{2} d c d \Omega$, и интегрирование по углам вследствие ортогональности полиномов Лежандра оставляет лишь проекцию ядра на $P_{0}$. В силу ОТГ, нижние индексы ненулевых ядер равны.

Свойство (12) позволяет найти еще два набора соотношений для ядер. Действительно, используя закон сохранения импульса $\mathbf{c}_{1}+\mathbf{c}_{2}=\mathbf{c}+\mathbf{c}^{\prime}$, можно записать

$$
\left(\mathbf{c}_{1}+\mathbf{c}_{2}\right) W\left(\mathbf{c}_{1}, \mathbf{c}_{2} \mid \mathbf{c}, \mathbf{c}^{\prime}\right)=\left(\mathbf{c}+\mathbf{c}^{\prime}\right) W\left(\mathbf{c}_{1}, \mathbf{c}_{2} \mid \mathbf{c}, \mathbf{c}^{\prime}\right) .
$$

С учетом (12) это соотношение можно переписать в виде

$$
\begin{aligned}
\mathbf{c}_{1} W\left(\mathbf{c}_{1}, \mathbf{c}_{2} \mid \mathbf{c}, \mathbf{c}^{\prime}\right) & +\mathbf{c}_{2} W\left(\mathbf{c}_{2}, \mathbf{c}_{1} \mid \mathbf{c}^{\prime}, \mathbf{c}\right)=\mathbf{c} W\left(\mathbf{c}_{1}, \mathbf{c}_{2} \mid \mathbf{c}, \mathbf{c}^{\prime}\right) \\
& +\mathbf{c}^{\prime} W\left(\mathbf{c}_{2}, \mathbf{c}_{1} \mid \mathbf{c}^{\prime}, \mathbf{c}\right) .
\end{aligned}
$$

Интегрируя (15) по с, $\mathbf{c}^{\prime}$, найдем

$$
\begin{aligned}
& \mathbf{c}_{1} \int G^{+}\left(\mathbf{c}, \mathbf{c}_{1}, \mathbf{c}_{2}\right) d \Omega c^{2} d c+\mathbf{c}_{2} \int G^{+}\left(\mathbf{c}, \mathbf{c}_{2}, \mathbf{c}_{1}\right) d \boldsymbol{\Omega} c^{2} d c \\
& =\int \mathbf{c} G^{+}\left(\mathbf{c}, \mathbf{c}_{1}, \mathbf{c}_{2}\right) d \Omega c^{2} d c+\int \mathbf{c} G^{+}\left(\mathbf{c}, \mathbf{c}_{2}, \mathbf{c}_{1}\right) d \Omega c^{2} d c
\end{aligned}
$$

Спроектируем это равенство на ось $z$, умножим его на $P_{i}\left(\cos \vartheta_{1}\right) P_{j}\left(\cos \vartheta_{2}\right)$ и проинтегрируем по $d \Omega_{1} d \Omega_{2}$. В результате получим

$$
\begin{aligned}
& c_{1} \int \cos \vartheta_{1} P_{i}\left(\cos \vartheta_{1}\right) P_{j}\left(\cos \vartheta_{2}\right) G^{+}\left(\mathbf{c}, \mathbf{c}_{1}, \mathbf{c}_{2}\right) d \mathbf{\Omega} d \mathbf{\Omega}_{1} d \mathbf{\Omega}_{2} c^{2} d c \\
& +c_{2} \int P_{i}\left(\cos \vartheta_{1}\right) \cos \vartheta_{2} P_{j}\left(\cos \vartheta_{2}\right) G^{+}\left(\mathbf{c}, \mathbf{c}_{2}, \mathbf{c}_{1}\right) d \mathbf{\Omega} d \mathbf{\Omega}_{1} d \mathbf{\Omega}_{2} \\
& \times c^{2} d c=\int c P_{1}(\cos \vartheta) P_{i}\left(\cos \vartheta_{1}\right) P_{j}\left(\cos \vartheta_{2}\right) G^{+}\left(\mathbf{c}, \mathbf{c}_{1}, \mathbf{c}_{2}\right) \\
& \times d \boldsymbol{\Omega} d \mathbf{\Omega}_{1} d \mathbf{\Omega}_{2} c^{2} d c+\int c P_{1}(\cos \vartheta) P_{i}\left(\cos \vartheta_{1}\right) P_{j}\left(\cos \vartheta_{2}\right) \\
& \times G^{+}\left(\mathbf{c}, \mathbf{c}_{2}, \mathbf{c}_{1}\right) d \boldsymbol{\Omega} d \mathbf{\Omega}_{1} d \mathbf{\Omega}_{2} c^{2} d c
\end{aligned}
$$

Учитывая, что

$$
x P_{i}(x)=\frac{i+1}{2 i+1} P_{i+1}(x)+\frac{i}{2 i+1} P_{i-1}(x)
$$

и выполняя интегрирование, найдем

$$
\begin{gathered}
\int c^{3}\left(G_{i, j}^{1+}\left(c, c_{1}, c_{2}\right)+G_{j, i}^{1+}\left(c, c_{2}, c_{1}\right)\right) d c \\
=c_{2} \frac{j+1}{2 j+1} \int G_{j+1, i}^{0+}\left(c, c_{2}, c_{1}\right) c^{2} d c \\
+c_{2} \frac{j}{2 j+1} \int G_{j-1, i}^{0+}\left(c, c_{2}, c_{1}\right) c^{2} d c \\
+c_{1} \frac{i+1}{2 i+1} \int G_{i+1, j}^{0+}\left(c, c_{1}, c_{2}\right) c^{2} d c \\
+c_{1} \frac{i}{2 i+1} \int G_{i-1, j}^{0+}\left(c, c_{1}, c_{2}\right) c^{2} d c
\end{gathered}
$$


Согласно ОТГ, ядра будут ненулевыми только в том случае, когда индексы различаются на единицу, $|i-j|=1$. Поэтому из последнего соотношения имеем

$$
\begin{gathered}
\int c^{3}\left(G_{i, i+1}^{1+}\left(c, c_{1}, c_{2}\right)+G_{i+1, i}^{1+}\left(c, c_{2}, c_{1}\right)\right) d c \\
=c_{2} \frac{i+1}{2 i+3} \int G_{i, i}^{0+}\left(c, c_{2}, c_{1}\right) c^{2} d c \\
+c_{1} \frac{i+1}{2 i+1} \int G_{i+1, i+1}^{0+}\left(c, c_{1}, c_{2}\right) c^{2} d c \\
\int c^{3}\left(G_{i, i+1}^{1+}\left(c, c_{2}, c_{1}\right)+G_{i+1, i}^{1+}\left(c, c_{1}, c_{2}\right)\right) d c \\
=c_{1} \frac{i+1}{2 i+3} \int G_{i, i}^{0+}\left(c, c_{1}, c_{2}\right) c^{2} d c \\
+c_{2} \frac{i+1}{2 i+1} \int G_{i+1, i+1}^{0+}\left(c, c_{2}, c_{1}\right) c^{2} d c .
\end{gathered}
$$

Используем теперь закон сохранения энергии $c_{1}^{2}+c_{2}^{2}=c^{2}+c^{\prime 2}$ совместно с (12). Это дает

$$
\begin{aligned}
c_{1}^{2} W\left(\mathbf{c}_{1}, \mathbf{c}_{2} \mid \mathbf{c}, \mathbf{c}^{\prime}\right) & +c_{2}^{2} W\left(\mathbf{c}_{2}, \mathbf{c}_{1} \mid \mathbf{c}^{\prime}, \mathbf{c}\right)=c^{2} W\left(\mathbf{c}_{1}, \mathbf{c}_{2} \mid \mathbf{c}, \mathbf{c}^{\prime}\right) \\
& +c^{\prime 2} W\left(\mathbf{c}_{2}, \mathbf{c}_{1} \mid \mathbf{c}^{\prime}, \mathbf{c}\right) .
\end{aligned}
$$

Интегрирование этого соотношения по с, $\mathbf{c}^{\prime}$ приводит к равенству

$$
\begin{aligned}
& c_{1}^{2} \int G^{+}\left(\mathbf{c}, \mathbf{c}_{1}, \mathbf{c}_{2}\right) d \mathbf{\Omega} c^{2} d c+c_{2}^{2} \int G^{+}\left(\mathbf{c}, \mathbf{c}_{2}, \mathbf{c}_{1}\right) d \mathbf{\Omega} c^{2} d c \\
& =\int c^{2} G^{+}\left(\mathbf{c}, \mathbf{c}_{1}, \mathbf{c}_{2}\right) d \mathbf{\Omega} c^{2} d c+\int c^{2} G^{+}\left(\mathbf{c}, \mathbf{c}_{2}, \mathbf{c}_{1}\right) d \mathbf{\Omega} c^{2} d c
\end{aligned}
$$

Умножая (17) на $P_{i}\left(\cos \vartheta_{1}\right) P_{j}\left(\cos \vartheta_{2}\right)$, интегрируя по $d \Omega_{1} d \Omega_{2}$ и используя ОТГ, получим

$$
\begin{gathered}
c_{1}^{2} \int G_{i, i}^{0+}\left(c, c_{1}, c_{2}\right) c^{2} d c+c_{2}^{2} \int G_{i, i}^{0+}\left(c, c_{2}, c_{1}\right) c^{2} d c \\
=\int\left(G_{i, i}^{0+}\left(c, c_{1}, c_{2}\right)+G_{i, i}^{0+}\left(c, c_{2}, c_{1}\right)\right) c^{4} d c .
\end{gathered}
$$

Используем теперь другое свойство симметрии функции $W\left(\mathbf{c}_{1}, \mathbf{c}_{2} \mid \mathbf{c}, \mathbf{c}^{\prime}\right)$. Учитывая закон сохранения импульса в столкновении, можно считать, что частица со скоростью $\mathbf{c}_{2}$ после столкновения приобретает скорость $\mathbf{c}_{1}+\mathbf{c}_{2}-\mathbf{c}$, а частица со скоростью $\mathbf{c}_{1}-$ скорость с. Это отражается в том, что выражение $W\left(\mathbf{c}_{2}, \mathbf{c}_{1} \mid \mathbf{c}_{1}+\mathbf{c}_{2}-\mathbf{c}, \mathbf{c}^{\prime}\right)$ в качестве второго сомножителя содержит $\delta\left(\frac{\mathbf{c}-\mathbf{c}^{\prime}}{2}\right)$. В то же время $W\left(\mathbf{c}_{1}, \mathbf{c}_{2} \mid \mathbf{c}, \mathbf{c}^{\prime}\right)$ содержит $\delta\left(\frac{\mathbf{c}_{1}+\mathbf{c}_{2}-\mathbf{c}-\mathbf{c}^{\prime}}{2}\right)$. Полагая $\mathbf{c}^{\prime}=\mathbf{c}$ в первом случае и $\mathbf{c}^{\prime}=\mathbf{c}_{1}+\mathbf{c}_{2}-\mathbf{c}$ во втором, найдем, что коэффициенты при $\delta$-функциях совпадают и равны

$$
\begin{aligned}
& v_{T} \tau_{T} n^{0} \sigma\left(\left|\mathbf{c}_{1}-\mathbf{c}_{2}\right| v_{T}, \arccos \left(\frac{\left(\left(\mathbf{c}_{1}-\mathbf{c}_{2}\right)\left(2 \mathbf{c}-\mathbf{c}_{1}-\mathbf{c}_{2}\right)\right)}{\left|\mathbf{c}_{1}-\mathbf{c}_{2}\right|\left|2 \mathbf{c}-\mathbf{c}_{1}-\mathbf{c}_{2}\right|}\right)\right) \\
& \quad \times \delta\left(\frac{\left(\mathbf{c}_{1}-\mathbf{c}_{2}\right)^{2}-\left(2 \mathbf{c}-\mathbf{c}_{1}-\mathbf{c}_{2}\right)^{2}}{2}\right) .
\end{aligned}
$$

Поэтому с учетом (9) имеем

$$
G^{+}\left(\mathbf{c}, \mathbf{c}_{1}, \mathbf{c}_{2}\right)=G^{+}\left(\mathbf{c}_{1}+\mathbf{c}_{2}-\mathbf{c}, \mathbf{c}_{2}, \mathbf{c}_{1}\right) .
$$

Проинтегрируем это равенство по направлениям скоростей $\mathbf{c}, \mathbf{c}_{2}, \mathbf{c}_{1}$. Слева получим $4 \pi G_{0,0}^{0+}\left(c, c_{1}, c_{2}\right)$. При интегрировании $G^{+}\left(\mathbf{c}_{1}+\mathbf{c}_{2}-\mathbf{c}, \mathbf{c}_{2}, \mathbf{c}_{1}\right)$ по направлению $\Omega$ скорости с в качестве оси сферической координатной системы выберем направление вектора $\mathbf{c}_{1}+\mathbf{c}_{2}$. Тогда если азимутальный угол $\boldsymbol{\Omega}$ равен $\varphi$, то азимутальный угол вектора $\mathbf{c}_{1}+\mathbf{c}_{2}-\mathbf{c}$ будет равен $\varphi-\pi$. Из простых геометрических соображений ясно, что

$$
c \cos \vartheta=\left|\mathbf{c}_{1}+\mathbf{c}_{2}\right|-\left|\mathbf{c}_{1}+\mathbf{c}_{2}-\mathbf{c}\right| \cos \vartheta^{\prime},
$$

где $\vartheta$ и $\vartheta^{\prime}-$ зенитные углы векторов $\Omega$ и $\mathbf{c}_{1}+\mathbf{c}_{2}-\mathbf{c}$ соответственно. Поскольку в силу закона сохранения энергии $\left(\mathbf{c}_{1}+\mathbf{c}_{2}-\mathbf{c}\right)^{2}=c_{1}^{2}+c_{2}^{2}-c^{2}$, то

$$
\begin{aligned}
& G^{+}\left(\mathbf{c}_{1}+\mathbf{c}_{2}-\mathbf{c}, \mathbf{c}_{2}, \mathbf{c}_{1}\right)= \\
& =G^{+}\left(\left(c_{1}^{2}+c_{2}^{2}-c^{2}\right)^{1 / 2}, \cos \vartheta^{\prime}, \varphi-\pi, \mathbf{c}_{2}, \mathbf{c}_{1}\right)
\end{aligned}
$$

Поэтому

$$
\begin{aligned}
& \iiint G^{+}\left(\mathbf{c}_{1}+\mathbf{c}_{2}-\mathbf{c}, \mathbf{c}_{2}, \mathbf{c}_{1}\right) d \mathbf{\Omega} d \mathbf{\Omega}_{1} d \mathbf{\Omega}_{2} \\
& =\iiint \int G^{+}\left(\left(c_{1}^{2}+c_{2}^{2}-c^{2}\right)^{1 / 2}, \cos \vartheta^{\prime}, \varphi-\pi, \mathbf{c}_{2}, \mathbf{c}_{1}\right) \\
& \times d \cos \vartheta d \varphi d \mathbf{\Omega}_{1} d \mathbf{\Omega}_{2}=\iiint \int G^{+}\left(\left(c_{1}^{2}+c_{2}^{2}-c^{2}\right)^{1 / 2},\right. \\
& \left.\cos \vartheta^{\prime}, \varphi, \mathbf{c}_{2}, \mathbf{c}_{1}\right) d \cos \vartheta^{\prime} d \varphi \frac{\left(c_{1}^{2}+c_{2}^{2}-c^{2}\right)^{1 / 2}}{c} d \mathbf{\Omega}_{1} d \mathbf{\Omega}_{2} \\
& =4 \pi \frac{\left(c_{1}^{2}+c_{2}^{2}-c^{2}\right)^{1 / 2}}{c} G_{0,0}^{0+}\left(\left(c_{1}^{2}+c_{2}^{2}-c^{2}\right)^{1 / 2}, c_{2}, c_{1}\right) .
\end{aligned}
$$

Таким образом:

$$
\begin{aligned}
& c G_{0,0}^{0+}\left(c, c_{1}, c_{2}\right)=\left(c_{1}^{2}+c_{2}^{2}-c^{2}\right)^{1 / 2} \\
& \quad \times G_{0,0}^{0+}\left(\left(c_{1}^{2}+c_{2}^{2}-c^{2}\right)^{1 / 2}, c_{2}, c_{1}\right) .
\end{aligned}
$$

Такой же результат был получен в работе [14], где рассматривалось кинетическое уравнение в изотропном случае. При этом в качестве независимой переменной вместо скорости использовалась энергия. Легко видеть, что аналогичное равенство можно получить для всех индексов $i$ :

$$
\begin{aligned}
c G_{i, i}^{0+}\left(c, c_{1}, c_{2}\right)= & \left(c_{1}^{2}+c_{2}^{2}-c^{2}\right)^{1 / 2} \\
& \times G_{i, i}^{0+}\left(\left(c_{1}^{2}+c_{2}^{2}-c^{2}\right)^{1 / 2}, c_{2}, c_{1}\right) .
\end{aligned}
$$

Отметим, что из (23) следует соотношение (18). Действительно, левую часть (18) можно представить 
в виде

$$
\begin{aligned}
& c_{1}^{2} \int G_{i i}^{0+}\left(c^{\prime}, c_{1}, c_{2}\right)\left(c^{\prime}\right)^{2} d c^{\prime}+c_{2}^{2} \int G_{i i}^{0+}\left(c^{\prime}, c_{2}, c_{1}\right)\left(c^{\prime}\right)^{2} d c^{\prime} \\
& =\frac{1}{2} \int\left(c_{1}^{2}+c_{2}^{2}-c^{2}\right) G_{i i}^{0+}\left(c, c_{1}, c_{2}\right) c^{2} d c \\
& +\frac{1}{2} \int\left(c_{1}^{2}+c_{2}^{2}-c^{2}\right) G_{i i}^{0+}\left(c, c_{2}, c_{1}\right) c^{2} d c \\
& +\frac{1}{2} \int c^{2} G_{i i}^{0+}\left(c, c_{1}, c_{2}\right) c^{2} d c+\frac{1}{2} \int c^{2} G_{i i}^{0+}\left(c, c_{2}, c_{1}\right) c^{2} d c .
\end{aligned}
$$

Здесь учтена симметрия $G_{i i}^{0+}\left(c^{\prime}, c_{1}, c_{2}\right)\left(c^{\prime}\right)^{2} d c^{\prime}$ относительно перестановки $c_{1}$ и $c_{2}$. Для преобразования первых членов в правой части (24) используем свойство (23) и проведем замену переменной $c^{\prime}=\left(c_{1}^{2}+c_{2}^{2}-c^{2}\right)^{1 / 2}$. Для первого члена это дает

$$
\begin{aligned}
& \frac{1}{2} \int\left(c_{1}^{2}+c_{2}^{2}-c^{2}\right) G_{i i}^{0+}\left(c, c_{1}, c_{2}\right) c^{2} d c \\
& =\frac{1}{2} \int\left(c_{1}^{2}+c_{2}^{2}-c^{2}\right)^{3 / 2} G_{i i}^{0+}\left(\left(c_{1}^{2}+c_{2}^{2}-c^{2}\right)^{1 / 2}, c_{2}, c_{1}\right) c d c \\
& =\frac{1}{2} \int\left(c^{\prime}\right)^{3} G_{i i}^{0+}\left(c^{\prime}, c_{2}, c_{1}\right) c^{\prime} d c^{\prime}
\end{aligned}
$$

Второй член приводится к такому же виду с точностью до перестановки $c_{1}$ и $c_{2}$, поэтому вся правая часть (24) равна $\int c^{4} G_{i i}^{0+}\left(c, c_{2}, c_{1}\right) d c+\int c^{4} G_{i i}^{0+}\left(c, c_{1}, c_{2}\right) d c$, что и означает справедливость (18).

Можно показать, что использование законов сохранения импульса и энергии совместно с рассматриваемым случаем симметрии не приводит к появлению новых соотношений для ядер.

Используем теперь принцип детального равновесия, согласно которому

$$
W\left(\mathbf{c}_{1}, \mathbf{c}_{2} \mid \mathbf{c}, \mathbf{c}^{\prime}\right)=W\left(\mathbf{c}, \mathbf{c}^{\prime} \mid \mathbf{c}_{1}, \mathbf{c}_{2}\right) .
$$

Если учесть, что величина относительной скорости не меняется при взаимодействии, то это равенство очевидно из (8). Используя (8) по аналогии с выводом свойства (19), найдем, что

$$
\int W\left(\mathbf{c}, \mathbf{c}^{\prime} \mid \mathbf{c}_{1}, \mathbf{c}_{2}\right) d \mathbf{c}^{\prime}=\int W\left(\mathbf{c}, \mathbf{c}_{1}+\mathbf{c}_{2}-\mathbf{c} \mid \mathbf{c}_{1}, \mathbf{c}^{\prime}\right) d \mathbf{c}^{\prime}
$$

Отсюда с учетом (25) имеем

$$
G^{+}\left(\mathbf{c}, \mathbf{c}_{1}, \mathbf{c}_{2}\right)=G^{+}\left(\mathbf{c}_{1}, \mathbf{c}, \mathbf{c}_{1}+\mathbf{c}_{2}-\mathbf{c}\right) .
$$

Как и раньше, проинтегрируем это равенство по направлениям скоростей $\mathbf{c}, \mathbf{c}_{1}, \mathbf{c}_{2}$ и примем во внимание, что вследствие сохранения энергии длина вектора $\mathbf{c}_{1}+\mathbf{c}_{2}-\mathbf{c}$ есть $\left(c_{1}^{2}+c_{2}^{2}-c^{2}\right)^{1 / 2}$. При интегрировании по $\Omega_{2}$ в качестве оси сферической координатной системы выберем направление вектора с-с 1 . Выразим сферические координаты $\left(\vartheta_{2}^{\prime}, \varphi_{2}^{\prime}\right)$ вектора $\mathbf{c}_{1}+\mathbf{c}_{2}-\mathbf{c}$ через сферические координаты $\left(\vartheta_{2}, \varphi_{2}\right)$ вектора $\mathbf{c}_{2}: \varphi_{2}^{\prime}=\varphi_{2}$,

$$
\cos \vartheta_{2}^{\prime}=\frac{c_{2} \cos \vartheta_{2}-\left|\mathbf{c}-\mathbf{c}_{1}\right|}{\left|\mathbf{c}_{1}+\mathbf{c}_{2}-\mathbf{c}\right|}
$$

Интеграл от правой части (26) можно привести к виду

$$
\begin{aligned}
& \iiint G^{+}\left(\mathbf{c}_{1}, \mathbf{c}, \mathbf{c}_{1}+\mathbf{c}_{2}-\mathbf{c}\right) d \boldsymbol{\Omega} d \mathbf{\Omega}_{1} d \mathbf{\Omega}_{2} \\
& =\iiint \int G^{+}\left(\mathbf{c}_{1}, \mathbf{c},\left(c_{1}^{2}+c_{2}^{2}-c^{2}\right)^{1 / 2}, \cos \vartheta_{2}^{\prime}, \varphi_{2}\right) d \cos \vartheta_{2} \\
& \times d \varphi_{2} d \boldsymbol{\Omega} d \mathbf{\Omega}_{1}=\iiint \int G^{+}\left(\mathbf{c}_{1}, \mathbf{c},\left(c_{1}^{2}+c_{2}^{2}-c^{2}\right)^{1 / 2},\right. \\
& \left.\cos \vartheta_{2}^{\prime}, \varphi_{2}\right) d \cos \vartheta_{2}^{\prime} d \varphi_{2} \frac{\left(c_{1}^{2}+c_{2}^{2}-c^{2}\right)^{1 / 2}}{c_{2}} d \boldsymbol{\Omega} d \mathbf{\Omega}_{1} \\
& =4 \pi \frac{\left(c_{1}^{2}+c_{2}^{2}-c^{2}\right)^{1 / 2}}{c_{2}} G_{0,0}^{0+}\left(c_{1}, c,\left(c_{1}^{2}+c_{2}^{2}-c^{2}\right)^{1 / 2}\right) .
\end{aligned}
$$

Все выкладки остаются справедливыми, если умножить (26) на полиномы Лежандра от $\cos \vartheta$ и $\cos \vartheta_{1}$. С учетом ОТГ для произвольных значений индексов $i$ имеем

$$
\begin{aligned}
c_{2} G_{i, 0}^{i+}\left(c, c_{1}, c_{2}\right)= & \left(c_{1}^{2}+c_{2}^{2}-c^{2}\right)^{1 / 2} \\
& \times G_{i, 0}^{i+}\left(c_{1}, c,\left(c_{1}^{2}+c_{2}^{2}-c^{2}\right)^{1 / 2}\right) .
\end{aligned}
$$

И в этом случае использование законов сохранения импульса и энергии совместно с (18) не дает новых соотношений для ядер.

Отметим еще связь между ядрами интегралов прямых и обратных столкновений. Как видно из (9), (10):

$$
G^{-}\left(\mathbf{c}, \mathbf{c}_{1}, \mathbf{c}_{2}\right)=\delta\left(\mathbf{c}_{1}-\mathbf{c}\right) \int G^{+}\left(\mathbf{c}^{\prime}, \mathbf{c}_{1}, \mathbf{c}_{2}\right) d \mathbf{c}^{\prime}
$$

Умножим теперь (29) на

$$
\frac{1}{2 \pi\left\|P_{l}\right\|^{2}} P_{l}(\cos \vartheta) P_{l_{1}}\left(\cos \vartheta_{1}\right) P_{l_{2}}\left(\cos \vartheta_{2}\right)
$$

и проинтегрируем по $d \boldsymbol{\Omega} d \boldsymbol{\Omega}_{1} d \boldsymbol{\Omega}_{2}$

$$
\begin{aligned}
& G_{l_{1} l_{2}}^{l-}\left(c, c_{1}, c_{2}\right)=\frac{1}{2 \pi\left\|P_{l}\right\|^{2}} \iiint G^{-}\left(\mathbf{c}, \mathbf{c}_{1}, \mathbf{c}_{2}\right) \\
& \times P_{l}(\cos \vartheta) P_{l_{1}}\left(\cos \vartheta_{1}\right) P_{l_{2}}\left(\cos \vartheta_{2}\right) d \mathbf{\Omega} d \mathbf{\Omega}_{1} d \mathbf{\Omega}_{2} \\
& =\frac{1}{2 \pi\left\|P_{l}\right\|^{2}} \iiint\left(\int G^{+}\left(\mathbf{c}^{\prime}, \mathbf{c}_{1}, \mathbf{c}_{2}\right) d \mathbf{c}^{\prime}\right) \\
& \times P_{l}(\cos \vartheta) P_{l_{1}}\left(\cos \vartheta_{1}\right) P_{l_{2}}\left(\cos \vartheta_{2}\right) \delta\left(\mathbf{c}_{1}-\mathbf{c}\right) d \Omega d \mathbf{\Omega}_{1} d \mathbf{\Omega}_{2} .
\end{aligned}
$$

Запишем $\delta$-функцию под интегралом в виде

$$
\delta\left(\mathbf{c}_{1}-\mathbf{c}\right)=\frac{\delta\left(c_{1}-c\right)}{c^{2}} \delta\left(\cos \vartheta_{1}-\cos \vartheta\right) \delta\left(\varphi_{1}-\varphi\right) .
$$


Интегрирование по $\Omega_{1}$ дает в правой части (30)

$$
\begin{aligned}
& \frac{\delta\left(c_{1}-c\right)}{c^{2}} \frac{1}{2 \pi\left\|P_{l}\right\|^{2}} \iiint\left(\int G^{+}\left(\mathbf{c}^{\prime}, \mathbf{c}, \mathbf{c}_{2}\right) c^{\prime 2} d c^{\prime}\right) \\
& \times P_{l}(\cos \vartheta) P_{l_{1}}(\cos \vartheta) P_{l_{2}}\left(\cos \vartheta_{2}\right) d \mathbf{\Omega}^{\prime} d \mathbf{\Omega} d \mathbf{\Omega}_{2} .
\end{aligned}
$$

Ясно, что произведение $P_{l}(\cos \theta) P_{l_{1}}(\cos \theta)$ может быть представлено в виде линейной комбинации полиномов Лежандра с индексами, не превосходящими $l+l_{1}$ :

$$
\begin{gathered}
P_{l}(x) P_{l_{1}}(x)=\sum_{k=0}^{l+l_{1}} C_{l_{1} k}^{l} P_{k}(x), \\
C_{l_{1} k}^{l}=\frac{1}{\left\|P_{k}\right\|^{2}} \int_{-1}^{1} P_{l}(x) P_{l_{1}}(x) P_{k}(x) d x .
\end{gathered}
$$

Поэтому

$G_{l_{1} l_{2}}^{l-}\left(c, c_{1}, c_{2}\right)=\frac{\delta\left(c_{1}-c\right)}{c^{2}} \sum_{k=0}^{l+l_{1}} C_{l_{1} k}^{l} \int G_{k l_{2}}^{0+}\left(c^{\prime}, c, c_{2}\right)\left(c^{\prime}\right)^{2} d c^{\prime}$.

Поскольку в силу ОТГ среди ядер $G_{k l_{2}}^{0+}\left(c^{\prime}, c, c_{2}\right)$ лишь ядро $G_{l_{2} l_{2}}^{0+}\left(c^{\prime}, c, c_{2}\right)$ отлично от нуля, то

$$
G_{l_{1} l_{2}}^{l-}\left(c, c_{1}, c_{2}\right)=\frac{\delta\left(c_{1}-c\right)}{c^{2}} C_{l_{1} l_{2}}^{l} \int G_{l_{2} l_{2}}^{0+}\left(c^{\prime}, c, c_{2}\right)\left(c^{\prime}\right)^{2} d c^{\prime} .
$$

Константы $C_{l_{1} l_{2}}^{l}$ отличны от нуля лишь при выполнении условий $l_{1} \leq l+l_{2}, l_{2} \leq l+l_{1}, l \leq l_{1}+l_{2}$, т. е. формула для ядра интеграла прямых столкновений согласуется с ОТГ.

Отметим, наконец, что, как видно из (11), для сечений рассеяния, симметричных относительно $\pi / 2$ (т.е. при выполнении условия $\sigma(\theta)=\sigma(\pi-\theta))$ ядра обладают дополнительным свойством симметрии - они оказываются симметричными относительно перестановки аргументов $c_{1}$ и $c_{2}$ (поскольку $\arccos x=\pi-\arccos (-x)$ ):

$$
G^{+}\left(\mathbf{c}, \mathbf{c}_{2}, \mathbf{c}_{1}\right)=G^{+}\left(\mathbf{c}, \mathbf{c}_{1}, \mathbf{c}_{2}\right) .
$$

При этом, естественно, выполняются равенства

$$
G_{l_{2} l_{1}}^{l+}\left(c, c_{2}, c_{1}\right)=G_{l_{1} l_{2}}^{l+}\left(c, c_{1}, c_{2}\right) .
$$

Соотношения (35), (36) справедливы, в частности, для изотропного рассеяния.

\section{Основное соотношение для ядер интеграла столкновений в случае взаимодействия частиц разных масс}

В работе [10] были получены связи между ядрами интеграла столкновений для случая взаимодействия частиц одинаковых масс. В этом разделе обобщим этот результат на случай частиц разных масс. Так же как и в [10], рассматриваем ФР в двух различных системах отсчета (базисах), движущихся с различными скоростями $\mathbf{u}_{0}, \mathbf{u}_{1}$ вдоль выделенной оси $z$. Пусть $\mathbf{c}_{a}, \mathbf{c}_{b}-$ безразмерные скорости частиц в базисе $\mathbf{u}_{0}$. Тогда скорости в базисе $\mathbf{u}_{1}$ будут

$$
\mathbf{c}_{a}^{\prime}=\mathbf{c}_{a}-\frac{\mathbf{u}_{1}-\mathbf{u}_{0}}{v_{T_{a}}}, \quad \mathbf{c}_{b}^{\prime}=\mathbf{c}_{b}-\frac{\mathbf{u}_{1}-\mathbf{u}_{0}}{v_{T_{b}}} .
$$

Учитывая, что $f_{i}\left(\mathbf{c}_{i}\right)=f_{i}^{\prime}\left(\mathbf{c}_{i}^{\prime}\right), i=a, b$, разложим ФР частиц $a$ и $b$ в базисах $\mathbf{u}_{0}$ и $\mathbf{u}_{1}$ по полиномам Лежандра

$$
\begin{aligned}
& \sum_{l=0}^{\infty} f_{l}^{a}\left(c_{a}\right) P_{l}\left(x_{a}\right)=\sum_{\lambda=0}^{\infty} F_{\lambda}^{a}\left(c_{a}^{\prime}\right) P_{l}\left(x_{a}^{\prime}\right), \\
& \sum_{l=0}^{\infty} f_{l}^{b}\left(c_{b}\right) P_{l}\left(x_{b}\right)=\sum_{\lambda=0}^{\infty} F_{\lambda}^{b}\left(c_{b}^{\prime}\right) P_{l}\left(x_{b}^{\prime}\right) .
\end{aligned}
$$

Здесь $x_{a}=\cos \vartheta_{a}, x_{b}=\cos \vartheta_{b}, x_{a}^{\prime}=\cos \vartheta_{a}^{\prime}, x_{b}^{\prime}=\cos \vartheta_{b}^{\prime}-$ косинусы углов между осью $z$ и векторами скоростей $\mathbf{c}_{a}, \mathbf{c}_{b}, \mathbf{c}_{a}^{\prime}, \mathbf{c}_{b}^{\prime}$, а скорости удовлетворяют соотношениям

$$
\begin{gathered}
c_{a}^{2}=c_{a_{z}}^{2}+c_{a_{\rho}}^{2}, \quad c_{a}^{\prime 2}=c_{a_{z}}^{\prime 2}+c_{a_{\rho}}^{\prime 2}, \\
c_{b}^{2}=c_{b_{z}}^{2}+c_{b_{\rho}}^{2}, \quad c_{b}^{\prime 2}=c_{b_{z}}^{\prime 2}+c_{b_{\rho}}^{\prime 2}, \\
c_{a_{z}}^{\prime}=c_{a_{z}}-\chi_{a}, \quad c_{a_{z}}=c_{a} x_{a}, \quad c_{a_{z}}^{\prime}=c_{a}^{\prime} x_{a}^{\prime}, \\
c_{b_{z}}^{\prime}=c_{b_{z}}-\chi_{b}, \quad c_{b_{z}}=c_{b} x_{b}, \quad c_{b_{z}}^{\prime}=c_{b}^{\prime} x_{b}^{\prime}, \\
\chi_{a}=\frac{\left|\mathbf{u}_{1}-\mathbf{u}_{0}\right|}{v_{T_{a}}}, \quad \chi_{b}=\frac{\left|\mathbf{u}_{1}-\mathbf{u}_{0}\right|}{v_{T_{b}}} .
\end{gathered}
$$

Скалярно умножая (40), (41) на полиномы Лежандра в базисе $\mathbf{u}_{1}$, т.е. на $P_{\lambda}\left(x_{a}^{\prime}\right), P_{\lambda}\left(x_{b}^{\prime}\right)$ соответственно, получим

$$
\begin{aligned}
& F_{\lambda}^{a}\left(c_{a}^{\prime}\right)=\sum_{l=0}^{\infty} \hat{\Psi}_{\lambda, l}^{a}\left(\chi_{a}, c_{a}^{\prime}\right) f_{l}^{a}\left(c_{a}\right), \\
& F_{\lambda}^{b}\left(c_{b}^{\prime}\right)=\sum_{l=0}^{\infty} \hat{\Psi}_{\lambda, l}^{b}\left(\chi_{b}, c_{b}^{\prime}\right) f_{l}^{b}\left(c_{b}\right),
\end{aligned}
$$

где операторы перехода $\hat{\Psi}_{\lambda, l}^{a}\left(\chi_{a}, c_{a}^{\prime}\right), \hat{\Psi}_{\lambda, l}^{b}\left(\chi_{b}, c_{b}^{\prime}\right)$ задаются формулами

$$
\hat{\Psi}_{\lambda, l}^{a}\left(\chi_{a}, c_{a}^{\prime}\right) f_{l}^{a}\left(c_{a}\right)=\frac{1}{\pi_{\lambda}} \int_{-1}^{1} P_{\lambda}\left(x_{a}^{\prime}\right) P_{l}\left(x_{a}\right) f_{l}^{a}\left(c_{a}\right) d x_{a}^{\prime},
$$$$
\hat{\Psi}_{\lambda, l}^{b}\left(\chi_{b}, c_{b}^{\prime}\right) f_{l}^{b}\left(c_{b}\right)=\frac{1}{\pi_{\lambda}} \int_{-1}^{1} P_{\lambda}\left(x_{b}^{\prime}\right) P_{l}\left(x_{b}\right) f_{l}^{b}\left(c_{b}\right) d x_{b}^{\prime} .
$$

Здесь $\pi_{\lambda}=\frac{2}{2 \lambda+1}-$ квадрат нормы полинома Лежандра $P_{\lambda}(x)$. Для обратных операторов $\hat{\Psi}_{l, \lambda}^{a}, \hat{\Psi}_{l, \lambda}^{b}$ имеем

$$
\hat{\Psi}_{l, \lambda}^{a}\left(-\chi_{a}, c_{a}\right) F_{\lambda}^{a}\left(c_{a}^{\prime}\right)=\frac{1}{\pi_{l}} \int_{-1}^{1} P_{l}\left(x_{a}\right) P_{\lambda}\left(x_{a}^{\prime}\right) F_{\lambda}^{a}\left(c_{a}^{\prime}\right) d x_{a},
$$




$$
\hat{\Psi}_{l, \lambda}^{b}\left(-\chi_{b}, c_{b}\right) F_{\lambda}^{b}\left(c_{b}^{\prime}\right)=\frac{1}{\pi_{l}} \int_{-1}^{1} P_{l}\left(x_{b}\right) P_{\lambda}\left(x_{b}^{\prime}\right) F_{\lambda}^{b}\left(c_{b}^{\prime}\right) d x_{b} .
$$

Действуя так же как в [10], для коэффициентов разложения безразмерного интеграла столкновений по $P_{\lambda}\left(x_{a}^{\prime}\right)$ в базисе $\mathbf{u}_{1}$ с учетом (7), (43), (45), (46) получим

$$
\begin{aligned}
& \int_{0}^{\infty} \int_{0}^{\infty} G_{\lambda_{1}, \lambda_{2}}^{\lambda}\left(c_{a}^{\prime}, c_{1 a}^{\prime}, c_{2 b}^{\prime}\right) F_{\lambda_{1}}^{a}\left(c_{a 1}^{\prime}\right) F_{\lambda_{2}}^{b}\left(c_{b 2}^{\prime}\right)\left(c_{a 1}^{\prime}\right)^{2} d c_{a 1}^{\prime}\left(c_{b 2}^{\prime}\right)^{2} \\
& \quad \times d c_{b 2}^{\prime}=\sum_{l, l_{1}, l_{2}} \int_{0}^{\infty} \int_{0}^{\infty} \hat{\Psi}_{\lambda, l}^{a}\left(\chi_{a}, c_{a}^{\prime}\right) G_{l_{1} l_{2}}^{l}\left(c_{a}, c_{a 1}, c_{b 2}\right) \\
& \quad \times \sum_{\lambda_{1} \lambda_{2}} \hat{\Psi}_{l_{1} \lambda_{1}}^{a}\left(-\chi_{a}, c_{a 1}\right) F_{\lambda_{1}}^{a}\left(c_{a 1}^{\prime}\right) \hat{\Psi}_{l_{2} \lambda_{2}}^{b}\left(-\chi_{b}, c_{b 2}\right) \\
& \quad \times F_{\lambda_{2}}^{b}\left(c_{b 2}^{\prime}\right)\left(c_{a 1}\right)^{2} d c_{a 1}\left(c_{b 2}\right)^{2} d c_{b 2} .
\end{aligned}
$$

Используя произвольность функций распределения $F_{\lambda_{1}}^{a}$, $F_{\lambda_{2}}^{b}$ после ряда преобразований, аналогичных описанным в [10], найдема

$$
\begin{aligned}
G_{\lambda_{1} \lambda_{2}}^{\lambda}\left(c_{a}^{\prime}, c_{a 1}^{\prime}, c_{b 2}^{\prime}\right)= & \sum_{l, l_{1}, l_{2}} \hat{\Psi}_{\lambda l}^{a}\left(\chi_{a}, c_{a}^{\prime}\right) \frac{\pi_{\lambda_{1}}}{\pi_{l_{1}}} \hat{\Psi}_{\lambda_{1} l_{1}}^{a}\left(\chi_{a}, c_{a 1}^{\prime}\right) \frac{\pi_{\lambda_{2}}}{\pi_{l_{2}}} \\
& \times \hat{\Psi}_{\lambda_{2} l_{2}}^{b}\left(\chi_{b}, c_{b 2}^{\prime}\right) G_{l_{1} l_{2}}^{l}\left(c_{a}, c_{a 1}, c_{b 2}\right) .
\end{aligned}
$$

Учтем теперь, что $\chi_{b}=\sqrt{m_{b} / m_{a}} \chi_{a}$, и продифференцируем это соотношение по $\chi_{a}$. При этом слева будет нуль, а справа возникнут производные только от операторов перехода. Полагая $\chi_{a}=0$, найдем

$$
\begin{aligned}
& \left.\sum_{l} \frac{d \hat{\Psi}_{\lambda l}^{a}\left(\chi_{a}, c_{a}^{\prime}\right)}{d \chi_{a}}\right|_{\chi_{a}=0} G_{\lambda_{1} \lambda_{2}}^{l}\left(c_{a}, c_{a 1}^{\prime}, c_{b 2}^{\prime}\right) \\
& +\left.\sum_{l_{1}} \frac{\pi_{\lambda_{1}}}{\pi_{l_{1}}} \frac{d \hat{\Phi}_{\lambda_{1} l_{1}}^{a}\left(\chi_{a}, c_{a 1}^{\prime}\right)}{d \chi_{a}}\right|_{\chi_{a}=0} G_{l_{1} \lambda_{2}}^{\lambda}\left(c_{a}, c_{a 1}, c_{b 2}\right) \\
& +\left.\sqrt{\frac{m_{b}}{m_{a}}} \sum_{l_{2}} \frac{\pi_{\lambda_{2}}}{\pi_{l_{2}}} \frac{d \hat{\Psi}_{\lambda_{2} l_{2}}^{a}\left(\chi_{b}, c_{b 2}^{\prime}\right)}{d \chi_{b}}\right|_{\chi_{a}=0} G_{\lambda_{1} l_{2}}^{\lambda}\left(c_{a}^{\prime}, c_{a 1}^{\prime}, c_{b 2}\right)=0 .
\end{aligned}
$$

В [10] было показано, что производная от оператора перехода имеет вид

$$
\begin{gathered}
\left.\frac{d \hat{\Psi}_{\lambda l}^{a}\left(\chi_{a}, c_{a}^{\prime}\right)}{d \chi_{a}}\right|_{\chi_{a}=0}=\delta_{l, \lambda-1} \hat{B}_{\lambda}^{(1)}\left(c_{a}\right)+\delta_{l, \lambda+1} \hat{B}_{\lambda}^{2}\left(c_{a}\right), \\
\hat{B}_{\lambda}^{(1)}\left(c_{a}\right)=\frac{1}{2 \lambda-1}\left(\frac{d}{d c_{a}}-\frac{\lambda-1}{c_{a}}\right), \\
\hat{B}_{\lambda}^{(2)}\left(c_{a}\right)=\frac{\lambda+1}{2 \lambda+3}\left(\frac{d}{d c_{a}}+\frac{\lambda+2}{c_{a}}\right) .
\end{gathered}
$$

Подставляя это выражение и аналогичное ему для

$$
\left.\frac{d \hat{\Psi}_{\lambda_{2} l_{2}}^{a}\left(\chi_{b}, c_{b 2}^{\prime}\right)}{d \chi_{b}}\right|_{\chi_{a}=0}
$$

в (47), заменяя $\lambda$ на $l, c_{a}^{\prime}$ на $c_{a}, c_{b}^{\prime}$ на $c_{b}$, окончательно получим

$$
\begin{aligned}
\hat{B}_{l}^{(1)}\left(c_{a}\right) G_{l_{1} l_{2}}^{l-1}\left(c_{a}, c_{a 1}, c_{b 2}\right)+\hat{B}_{l}^{(2)}\left(c_{a}\right) G_{l_{1} l_{2}}^{l+1}\left(c_{a}, c_{a 1}, c_{b 2}\right) \\
\quad+\hat{B}_{l_{1}}^{(3)}\left(c_{a 1}\right) G_{l_{1}+1, l_{2}}^{l}\left(c_{a}, c_{a 1}, c_{b 2}\right) \\
\quad+\hat{B}_{l_{1}}^{(4)}\left(c_{a 1}\right) G_{l_{1}-1, l_{2}}^{l}\left(c_{a}, c_{a 1}, c_{b 2}\right) \\
\quad+\sqrt{\frac{m_{b}}{m_{a}}}\left(\hat{B}_{l_{2}}^{(3)}\left(c_{b 2}\right) G_{l_{1}, l_{2}+1}^{l}\left(c_{a}, c_{a 1}, c_{b 2}\right)\right. \\
\left.+\hat{B}_{l_{2}}^{(4)}\left(c_{b 2}\right) G_{l_{1}, l_{2}-1}^{l}\left(c_{a}, c_{a 1}, c_{b 2}\right)\right)=0 .
\end{aligned}
$$

Здесь $\hat{B}_{l}^{(1)}\left(c_{a}\right), \hat{B}_{l}^{(2)}\left(c_{a}\right)$ задаются формулами (48),

$$
\begin{aligned}
& \hat{B}_{l}^{(3)}\left(c_{a}\right)=\frac{l+1}{2 l+1}\left(\frac{d}{d c_{a}}+\frac{l+2}{c_{a}}\right), \\
& \hat{B}_{l}^{(4)}\left(c_{a}\right)=\frac{1}{2 l+1}\left(\frac{d}{d c_{a}}-\frac{l-1}{c_{a}}\right) .
\end{aligned}
$$

Таким образом, как и в случае одинаковых частиц, связи (49) можно рассматривать как рекуррентные соотношения, позволяющие построить любое нелинейное ядро, если известно ядро $G_{0,0}^{0}\left(c_{a}, c_{a 1}, c_{b 2}\right)$. Отметим, что связи (49) могут быть получены и с помощью соотношений между матричными элементами интеграла столкновений в случае частиц разных масс. Эти соотношения приведены в [8]. Используя представление ядер через матричные элементы, соотношения между матричными элементами и рекуррентные соотношения между полиномами Сонина можно получить (49).

\section{Инварианты интеграла столкновений и свойства ядер}

Рассмотрим законы сохранения для газа, состоящего из частиц разных сортов. Уравнение Больцмана для компонента газовой смеси в отсутствие внешних сил можно записать в форме

$$
\left(\frac{\partial}{\partial t}+\mathbf{v}_{a} \frac{\partial}{\partial r}\right) n_{a}(r, t) f_{a}\left(\mathbf{v}_{a}, r, t\right)=\sum_{b} n_{a} n_{b} \hat{I}\left(f_{a}, f_{b}\right) .
$$

Число частиц сорта сохраняется при взаимодействии с частицами любого другого сорта, поэтому интеграл по скоростям $\mathbf{v}_{a}$ от $\hat{I}\left(f_{a}, f_{b}\right)$ равен нулю. В безразмерных переменных это условие запишется в виде

$$
\iiint G\left(\mathbf{c}_{a}, \mathbf{c}_{a 1}, \mathbf{c}_{b 2}\right) f_{a}\left(\mathbf{c}_{a 1}\right) f_{b}\left(\mathbf{c}_{b 2}\right) d \mathbf{c}_{a 1} d \mathbf{c}_{b 2} d \mathbf{c}_{a}=0 .
$$

Интегрируя по скоростям $\mathbf{c}_{a}$ разложение интеграла столкновений (7), находим

$$
\begin{gathered}
\sum_{l_{1} l_{2}} \int_{0}^{\infty} \int_{0}^{\infty} \int_{0}^{\infty} G_{l_{1} l_{2}}^{0}\left(c_{a}, c_{a 1}, c_{b 2}\right) f_{l_{1}}^{a}\left(c_{a 1}\right) f_{l_{2}}^{b}\left(c_{b 2}\right)\left(c_{a 1}\right)^{2} \\
\times d c_{a 1}\left(c_{b 2}\right)^{2} d c_{b 2}\left(c_{a}\right)^{2} d c_{a}=0
\end{gathered}
$$


Поскольку в соответствии с ОТГ ядра $G_{l_{1} l_{2}}^{0}$ отличны от нуля лишь при равенстве нижних индексов, то вследствие произвольности $Ф$ Р частиц сортов $a$ и $b$ должно выполняться условие

$$
\int_{0}^{\infty} G_{l_{1} l_{1}}^{0}\left(c_{a}, c_{a 1}, c_{b 2}\right)\left(c_{a}\right)^{2} d c_{a}=0 .
$$

Для однокомпонентного газа соотношение (50) с учетом ОТГ переходит в

$$
\begin{aligned}
& \sum_{l_{1}} \int_{0}^{\infty} \int_{0}^{\infty} \int_{0}^{\infty} G_{l_{1} l_{1}}^{0}\left(c, c_{1}, c_{2}\right) f_{l_{1}}\left(c_{1}\right) f_{l_{1}}\left(c_{2}\right)\left(c_{1}\right)^{2} \\
& \quad \times d c_{1}\left(c_{2}\right)^{2} d c_{2}(c)^{2} d c=0 .
\end{aligned}
$$

Вследствие произвольности $f_{l_{1}}(c)$ получим

$$
\int_{0}^{\infty} G_{l_{1} l_{1}}^{0}\left(c, c_{1}, c_{2}\right)(c)^{2} d c+\int_{0}^{\infty} G_{l_{1} l_{1}}^{0}\left(c, c_{2}, c_{1}\right)(c)^{2} d c=0 .
$$

Свойство (14) позволяет утверждать, что (51) должно выполняться и в случае однокомпонентного газа.

Законы сохранения энергии и импульса не выполняются для каждой компоненты по отдельности. Поэтому рассмотрим однокомпонентный газ. Закон сохранения энергии с учетом (7) запишется

$$
\begin{aligned}
& \sum_{l_{1}, l_{2}} \int_{0}^{\infty} \int_{0}^{\infty} \int_{0}^{\infty} G_{l_{1} l_{2}}^{0}\left(c, c_{1}, c_{2}\right) f_{l_{1}}\left(c_{1}\right) f_{l_{2}}\left(c_{2}\right)\left(c_{1}\right)^{2} \\
& \quad \times d c_{1}\left(c_{2}\right)^{2} d c_{2}(c)^{4} d c=0 .
\end{aligned}
$$

Поэтому, в силу произвольности ФР и справедливости ОТГ, ядра должны удовлетворять соотношениям

$$
\int_{0}^{\infty} G_{l_{1} l_{1}}^{0}\left(c, c_{1}, c_{2}\right)(c)^{4} d c+\int_{0}^{\infty} G_{l_{1} l_{1}}^{0}\left(c, c_{2}, c_{1}\right)(c)^{4} d c=0 .
$$

Закон сохранения импульса рассмотрим в осесимметричном случае. Для этого умножаем (7) на $c_{z}=c \cos \theta$ и интегрируем по $d^{3} c$. Это дает

$$
\begin{aligned}
& \sum_{l_{1}, l_{2}} \int_{0}^{\infty} \int_{0}^{\infty} \int_{0}^{\infty} G_{l_{1} l_{2}}^{1}\left(c, c_{1}, c_{2}\right) f_{l_{1}}\left(c_{1}\right) f_{l_{2}}\left(c_{2}\right)\left(c_{1}\right)^{2} \\
& \quad \times d c_{1}\left(c_{2}\right)^{2} d c_{2}(c)^{3} d c=0 .
\end{aligned}
$$

С учетом ОТГ и произвольности ФР получаем, что ядра должны удовлетворять условиям

$$
\int_{0}^{\infty} G_{l_{1}, l_{1}+1}^{1}\left(c, c_{1}, c_{2}\right)(c)^{3} d c+\int_{0}^{\infty} G_{l_{1}+1, l_{1}}^{1}\left(c, c_{2}, c_{1}\right)(c)^{3} d c=0 .
$$

Отметим, что свойства ядер, полученные в первом разделе, гарантируют выполнение условий (51)-(53). Так, интегрируя (34), при $l=0$ легко получить

$$
\begin{aligned}
& \int G_{l_{1} l_{1}}^{0-}\left(c, c_{1}, c_{2}\right) c^{2} d c=C_{l_{1} l_{1}}^{0} \int G_{l_{1} l_{1}}^{0+}\left(c^{\prime}, c_{1}, c_{2}\right)\left(c^{\prime}\right)^{2} d c^{\prime} \\
& =\int G_{l_{1} l_{1}}^{0+}\left(c^{\prime}, c_{1}, c_{2}\right)\left(c^{\prime}\right)^{2} d c^{\prime}
\end{aligned}
$$

в полном соответствии с (51). Для доказательства справедливости (52) опять используем (34). Подстановка (34) при $l=0$ в (52) дает

$$
\begin{aligned}
& \int G_{l_{1} l_{1}}^{0-}\left(c, c_{1}, c_{2}\right) c^{4} d c+\int G_{l_{1} l_{1}}^{0-}\left(c, c_{2}, c_{1}\right) c^{4} d c \\
& =c_{1}^{2} \int G_{l_{1} l_{1}}^{0+}\left(c^{\prime}, c_{1}, c_{2}\right)\left(c^{\prime}\right)^{2} d c^{\prime} \\
& +c_{2}^{2} \int G_{l_{1} l_{1}}^{0+}\left(c^{\prime}, c_{2}, c_{1}\right)\left(c^{\prime}\right)^{2} d c^{\prime} .
\end{aligned}
$$

Согласно (18), выражение в правой части равно

$$
\int\left(G_{l_{1} l_{1}}^{0+}\left(c, c_{1}, c_{2}\right)+G_{l_{1} l_{1}}^{0+}\left(c, c_{2}, c_{1}\right)\right) c^{4} d c
$$

что и означает выполнение (52).

Соотношение (34) при $l=1$ позволяет получить и условие (53). Действительно,

$$
\begin{aligned}
G_{l_{1}, l_{1}+1}^{1-}\left(c, c_{1}, c_{2}\right)= & \frac{\delta\left(c_{1}-c\right)}{c_{1}^{2}} C_{l_{1}, l_{1}+1}^{1} \\
& \times \int G_{l_{1}+1, l_{1}+1}^{0+}\left(c^{\prime}, c, c_{2}\right)\left(c^{\prime}\right)^{2} d c^{\prime}, \\
G_{l_{1}+1, l_{1}}^{1-}\left(c, c_{2}, c_{1}\right)= & \frac{\delta\left(c_{2}-c\right)}{c_{2}^{2}} C_{l_{1}+1, l_{1}}^{1} \\
& \times \int G_{l_{1}, l_{1}}^{0+}\left(c^{\prime}, c, c_{1}\right)\left(c^{\prime}\right)^{2} d c^{\prime} .
\end{aligned}
$$

Умножая эти равенства на $c^{3}$ и интегрируя по $c$, найдем

$$
\begin{gathered}
\int\left(G_{l_{1}, l_{1}+1}^{1-}\left(c, c_{1}, c_{2}\right)+G_{l_{1}+1, l_{1}}^{1-}\left(c, c_{2}, c_{1}\right)\right) c^{3} d c \\
=c_{1} C_{l_{1}, l_{1}+1}^{1} \int G_{l_{1}+1, l_{1}+1}^{0+}\left(c^{\prime}, c_{1}, c_{2}\right)\left(c^{\prime}\right)^{2} d c^{\prime} \\
+c_{2} C_{l_{1}+1, l_{1}}^{1} \int G_{l_{1}, l_{1}}^{0+}\left(c^{\prime}, c_{2}, c_{1}\right)\left(c^{\prime}\right)^{2} d c^{\prime} .
\end{gathered}
$$

Поскольку

$$
C_{l, l+1}^{1}=\frac{l+1}{2 l+1}, \quad C_{l+1, l}^{1}=\frac{l+1}{2 l+3},
$$

правая часть этого соотношения совпадает с правой частью (16), и, следовательно:

$$
\begin{aligned}
& \int\left(G_{l_{1}, l_{1}+1}^{1-}\left(c, c_{1}, c_{2}\right)+G_{l_{1}+1, l_{1}}^{1-}\left(c, c_{2}, c_{1}\right)\right) c^{3} d c \\
& =\int c^{3}\left(G_{l_{1}, l_{1}+1}^{1+}\left(c, c_{1}, c_{2}\right)+G_{l_{1}+1, l_{1}}^{1+}\left(c, c_{2}, c_{1}\right)\right) d c,
\end{aligned}
$$

т.е. (53) выполняется. 


\section{Заключение}

Исследованы общие свойства ядер интегральных операторов, представляющих собой проекции интеграла столкновений на полиномы Лежандра. Показано, что из симметрии парных столкновений, из законов сохранения энергии и импульса в парном столкновении и из принципа детального равновесия следует ряд соотношений между ядрами, которые справедливы для любого сечения рассеяния. В случае симметричных сечений рассеяния появляются дополнительные свойства, которые могут быть использованы при расчете ядер. Ранее нами были получены соотношения между ядрами с разными индексами (индексы ядер соответствуют индексам полиномов Лежандра, на которые проецируется интеграл столкновений) в случае взаимодействия частиц с равными массами, и на их основе была построена процедура последовательного определения ядер. Эти соотношения обобщены на случай взаимодействия частиц с разными массами. Показано, что полученные свойства ядер обеспечивают аддитивную инвариантность энергии, импульса и числа частиц.

\section{Конфликт интересов}

Авторы заявляют, что у них нет конфликта интересов.

\section{Список литературы}

[1] Burnett D. // Proc. London Math. Soc. 1935. Vol. 39. P. 385-430.

[2] Burnett D. // Proc. London Math. Soc. 1935. Vol. 40. P. $382-435$.

[3] Grad H. // Comm. Pure Appl. Math. 1949. Vol. 2. P. 311.

[4] Эндер А.Я., Эндер И.А. Интеграл столкновений уравнения Больцмана и моментный метод. СПб.: Изд-во Санкт-Петербургского ун-та, 2003. 224 с.

[5] Ender A.Ya., Ender I.A. // Phys. Fluids. 1999. Vol. 11. P. 2720-2730.

[6] Ender A.Ya., Ender I.A. // Transport Theor. Stat. Phys. 2007. Vol. 56. P. 563-588.

[7] Ender A.Ya., Ender I.A., Gerasimenko A.B. // Open Plasm. Phys. J. 2009. Vol. 2. P. 24-62.

[8] Эндер И.А., Бакалейников Л.А., Флегонтова Е.Ю., Герасименко А.Б. // ЖТФ. 2017. Т. 87. Вып. 8. С. 1136-1143.

[9] Эндер И.А., Бакалейников Л.А., Флегонтова Е.Ю., Герасименко А.Б. // ЖТФ. 2017. Т. 87. Вып. 9. С. 1297-1302.

[10] Эндер А.Я., Эндер И.А., Бакалейников Л.А. // ДАН. 2011. Т. 437. Вып. 5. С. 621-623.

[11] Ender A.Ya., Ender I.A., Bakaleinikov L.A., Flegontova E.Yu. // Europ. J. Mech. B. Fluids. 2012. Vol. 36. P. 17-24.

[12] Бакалейников Л.А., Флегонтова Е.Ю., Эндер А.Я., Эндер И.А. // ЖТФ. 2016. Т. 86. Вып. 4. С. 10-20.

[13] Бакалейников Л.А., Тропп Э.А., Флегонтова Е.Ю., Эндер И.А. // ЖТФ. 2018. Т. 88. Вып. 10. С. 1492-1501.

[14] Kugerl G., Schurrer F. // J. Appl. Math. Phys. (ZAMP). 1991. Vol. 42. P. 362-369.
[15] Эндер А.Я., Эндер И.А. Интегральное преобразование уравнения Больцмана для различных законов взаимодействия частиц. Препринт ФТИ РАН № 605. Л. 1979. 54 с.

[16] Черемисин Ф.Г. // ЖВММФ. 2006. Т. 46. Вып. 2. С. 329 343.

[17] Чепмен С., Каулинг T. Математическая теория неоднородных газов. М.: ИИЛ, 1960. $510 \mathrm{c}$.

[18] Ферцигер Джс., Капер Г. Математическая теория процессов переноса в газах. М.: Мир, 1976. 554 с.

[19] Вальдман Л. Явления переноса в газах при среднем давлении. В кн.: Термодинамика газов. М.: Машиностроение, 1970. С. 169-414.

[20] Бакалейников Л.А., Тропп Э.А., Флегонтова Е.Ю., Эндер А.Я., Эндер И.А. // ЖТФ. 2015. Т. 85. Вып. 1. С. 10-14. 